\title{
CONFIABILIDAD DE NUEVOS HÍBRIDOS DE MAÍZ, EN PANAMÁ1
}

\author{
Ismael Camargo ${ }^{2}$, Román Gordón ${ }^{2}$, Jorge Franco ${ }^{2}$, Andrés González ${ }^{2}$, Eric Quirós ${ }^{2}$, Alejandro Figueroa ${ }^{2}$
}

\section{RESUMEN}

Confiabilidad de nuevos híbridos de maíz, en Panamá. El presente estudio se efectuó para estimar la confiabilidad de los híbridos más sembrados en el país; para ello, se utilizó una metodología que determina la confiabilidad de la respuesta $\left(\mathrm{RN}_{\mathrm{i}}\right.$ o probabilidad de respuesta normalizada superior a cero) de los cultivares superiores con respecto a un cultivar testigo. Se determinó la confiabilidad de la respuesta de los híbridos: 3018, 3031, HS-8, P-8916, P-9422, P-9490 y X-1358K contra el testigo X-304C. Los híbridos evaluados se clasificaron en tres categorías con respecto al testigo: Genotipos de confiabilidad buena (HS-8, P-9490 y X-1358K, $\left.0,8 \geq \mathrm{RN}_{\mathrm{i}}<0,9\right)$, confiabilidad promedio $(3018,3031, \mathrm{P}-9422$, $\left.0,6 \geq \mathrm{RN}_{\mathrm{i}}<0,8\right)$ y confiabilidad baja $\left(\mathrm{P}-8916 ; 0,5 \geq \mathrm{RN}_{\mathrm{i}}<0,6\right)$. Los valores RNi para cada genotipo representan la probabilidad de que un genotipo supere en promedio el nivel de producción establecido por el híbrido testigo X-304C. Un RNi de 0,8 significa que en 8 de cada 10 casos (localidades) hubo una respuesta superior del nuevo genotipo con respecto al testigo y por lo tanto su recomendación es viable. Por otro lado, se verificó que la confiabilidad es una medida estrechamente relacionada a parámetros de estabilidad basados en modelos de regresión. De acuerdo al estudio, los híbridos que presentaron un comportamiento superior con relación al testigo fueron: HS-8, P-9490 y X-1358K que combinaron alto potencial de rendimiento y amplia adaptabilidad en los diferentes ambientes y años.

\begin{abstract}
Confidence for recommending new corn hybrids in Panama. The corn-breeding project of IDIAP evaluated 96 corn cultivars in 132 localities, during 1989-2000. The highest yielding genotypes have been recommended to the growers. Nevertheless, to continue improving the evaluation system of new cultivars, it is necessary to determine their confidence degree in relation to the regional cultivars. The present study aimed to estimate the stability of the most sown hybrids in the country by determining the performance, normalised over zero ( $\mathrm{RNi}$ ) of the superior cultivars. The confidence of performance was determined for the hybrids: 3018, 3031, HS-8, P-8916, P-94922, P-9490 and X-1358K; compared to the hybrid X-304C. The evaluated hybrids were classified in three categories: Genotypes with high confidence (HS-8, P-9490 y X-1358K; $0.8=\mathrm{RNi}<0.9$ ), medium confidence $(3018,3031$, P-9422; $0.6=\mathrm{RNi}<0.8)$ and low confidence $(\mathrm{P}-8916 ; 0.5=\mathrm{RNi}<0.6)$. The values $\mathrm{RNi}$ for each genotype represent the probability that a genotype exceeds the mean level of production of the reference hybrid (X$304 \mathrm{C}$ ), RNi of 0.8 means that the performance of the new hybrids was superior in 8 of 10 localities. Additionally, it was verified that the confidence is a measure closely related to parameters of stability based on a regression model. This study stated that hybrids with superior capabilities of production and adaptation to diverse ecological zones through the years were HS-8, P-9490 and X-1358K.
\end{abstract}

\section{INTRODUCCIÓN}

El proyecto de mejoramiento genético de maíz del IDIAP, evaluó en el período 1989-2000, 96 cultivares de maíz en 118 localidades. De éstos, los genotipos con mejor comportamiento han sido recomendados para su comercialización y siembra en las zonas maiceras. No obstante, para continuar perfeccionando el sistema de evaluación y liberación de nuevos cultivares, es necesario determinar el grado de confiabilidad de los nuevos genotipos con relación al cultivar testigo.

El ensayo denominado Prueba Regional de Maíz (PRM), permite la evaluación sistemática en ambientes

1 Recibido para publicación el 2 de abril del 2001.Presentado en la XLVII Reunión Anual del PCCMCA. San José, Costa Rica, 2001.

2 Programa de Maíz, Instituto de Investigación Agropecuaria de Panamá (IDIAP), Apdo. 6-4391, Estafeta El Dorado, Panamá 6A, Panamá. Tel: (507) 9933253. E-mail: icamargo@cwpanama.net. 
contrastantes de los genotipos generados por el programa nacional, y las empresas privadas que comercializan semilla en el país. Este ensayo ha demostrado ser una herramienta efectiva para la evaluación experimental de los mejores híbridos disponibles, también ha permitido demostrar que los híbridos de maíz generados a través de la colaboración horizontal IDIAP-CIMMYTPRM, son competitivos con los materiales desarrollados por las empresas privadas.

Utilizando la base de datos histórica de la Prueba Regional 1989-2000, se determinó que en este período se realizó la evaluación de 96 genotipos en 121 localidades, siendo el testigo el cultivar X-304C. Los resultados experimentales a través de los años han mostrado la superioridad en rendimiento de los nuevos híbridos sobre el testigo histórico. Con base en estos resultados, 22 de ellos, han sido recomendados para su comercialización; sin embargo, no se han llevado a cabo estudios que permitan cuantificar si las magnitudes de las diferencias de rendimientos respecto al testigo, representan un progreso genético en rendimiento, en adaptabilidad y estabilidad de los nuevos cultivares con relación al testigo.

Jones (1988) propuso una metodología que permitía la comparación de los cultivares contra el testigo utilizando los datos de varios años. Eskridge y Mumm (1992), Eskridge et al. (1993) proponen el análisis de confiabilidad basados en las diferencias de rendimiento de un híbrido respecto al testigo adaptado. Córdova et al. (1993), utilizando datos experimentales del ensayo uniforme del PCCMCA (1988-1990), demostró la aplicación práctica de la metodología para determinar la confiabilidad de las ganancias en rendimiento sobre el testigo regional $\mathrm{H} 5$. El análisis de confiabilidad propuesto por Eskridge y Mumm (1992), Eskridge et al. (1993) es similar, en principio, a la medición de superioridad propuesto por Lin y Binns (1994). Por otro lado, Baker (1996), indica que la medida de superioridad y el análisis de confiabilidad representan avances importantes en el entendimiento de los problemas del mejoramiento genético, producto de la interacción genotipo-ambiente.

Los objetivos del presente estudio son: cuantificar la confiabilidad de las ganancias en rendimiento de siete híbridos de maíz en comparación con el testigo X304C y verificar si la función de confiabilidad está relacionada con la estabilidad de los genotipos evaluados respecto al testigo.

\section{MATERIALES Y MÉTODOS}

\section{Origen de los datos experimentales}

Los datos experimentales utilizados en este estudio fueron obtenidos de la base de datos correspondiente a la Prueba Regional de Maíz del Instituto de Investigación Agropecuaria de Panamá (IDIAP) durante los últimos 12 años. En el Cuadro 1, se observan los ocho híbridos considerados en el estudio, además de su origen, número de localidades, años de evaluación y tipo de híbrido. Los genotipos seleccionados representan los materiales más utilizados en las siembras comerciales de maíz en Panamá, durante el período 1989-2000. Para realizar el análisis de confiabilidad con un nivel de precisión aceptable, Eskridge et al. (1993), sugieren un mínimo de 15 ambientes diferentes para efectuar la comparación con el testigo. Por otro lado, como esta metodología permite la comparación de diferentes cultivares con un testigo común, la selección del testigo adecuado puede tener un impacto importante en la estimación de la confiabilidad.

Cuadro 1. Híbridos considerados en el estudio, origen, número de localidades y años de evaluación. Panamá, 19892000 .

\begin{tabular}{llrccc}
\hline Híbridos & $\begin{array}{c}\text { Institución/ } \\
\text { empresas }\end{array}$ & $\begin{array}{c}\mathbf{N}^{\circ} \text { de } \\
\text { local }\end{array}$ & $\begin{array}{c}\mathbf{N}^{\circ} \text { de } \\
\text { años }\end{array}$ & $\begin{array}{c}\text { Tipo de } \\
\text { hib. }\end{array}$ & $\begin{array}{c}\text { Año } \\
\text { liberación }\end{array}$ \\
\hline 3018 & Pioneer & 80 & 8 & Doble & 1995 \\
3031 & Pioneer & 37 & 4 & Doble & 1995 \\
X-1358K & Pioneer & 38 & 4 & Simple & 1999 \\
HS-8 & Cristiani & 27 & 3 & Triple & 1999 \\
& Brurkard & & & & \\
P-8916 & IDIAP & 111 & 10 & Triple & 1990 \\
P-9422 & IDIAP & 55 & 6 & Triple & 1999 \\
P-9490 & IDIAP & 55 & 6 & Triple & 1999 \\
X-304C-T & Pioneer & 121 & 11 & Simple & 1996 \\
\hline
\end{tabular}

\section{Confiabilidad estadística de las respuestas}

Según Eskridge et al. (1993), la confiabilidad estadística de la respuesta en rendimiento del i-écimo genotipo se determina a partir de los diferenciales de rendimiento(di) con respecto al testigo, o sea:

$$
d_{i}=P\left(Y_{i}-Y_{c}>d_{i}\right)
$$

donde:

$\mathbf{P}($.) representa la probabilidad, $\mathbf{Y}_{\mathbf{i}}$ respuesta del híbrido evaluado 
$\mathbf{Y}_{\mathbf{c}}$ respuesta del testigo, y

$\mathbf{d}_{\mathbf{i}}$ es la diferencia entre ambos.

Córdova et al. (1993) indican que las hipótesis alternas planteadas en este estudio se refieren, a si el valor promedio de di es mayor o menor que cero es decir:

$$
\begin{array}{ll}
\text { Ha: } & d_{i} \leq 0 \\
\text { Ha: } & d_{i}>0
\end{array}
$$

Para probar las hipótesis se calculan primero los valores promedios de di y la desviación estándar de las diferencias a través de todas las localidades $\left(\mathrm{S}_{\mathrm{di}}\right)$. Posteriormente se determina el valor estandarizado $\left(\mathrm{d}_{\mathrm{i}} /\right.$ $\mathrm{S}_{\mathrm{di}}$ ) al cual se le determina la probabilidad normal estandarizada (usando la tabla de $\mathrm{Z}$ normal de una cola) A esta probabilidad se le denomina Confiabilidad Normalizada con respecto al testigo $\left(\mathrm{RN}_{\mathrm{i}}\right)$. El análisis de confiabilidad se calculó para cada comparación de interés, luego se procedió a ordenar de manera ascendente los genotipos en función de esta probabilidad (Córdova et al. 1993).

El valor de $\mathrm{RN}_{\mathrm{i}}$ para cada genotipo representa la probabilidad de que un genotipo específico supere en promedio el nivel de producción establecido por el híbrido testigo (X-304C), en el conjunto de todos los sitios y años donde se efectuaron las evaluaciones. De acuerdo a Córdova et al. (1993), los genotipos se clasifican en las siguientes categorías según el $\mathrm{RN}_{\mathrm{i}}$ estimado: a)confiabilidad superior de $0,9 \geq \mathrm{RNi}<1,0$; b) buena de $0,8 \geq \mathrm{RNi}<0,9 ;$ c) promedio de $0,6 \geq \mathrm{RNi}<0,8$ y; d) baja por debajo de 0,6. Entre tanto, Eskridge et al. (1993) sugiere realizar la prueba Q de Cochran 1950 para separar los valores de confiabilidad de acuerdo al nivel de significancia.

\section{Estabilidad relativa de los genotipos}

La interacción genotipo-ambiente merece gran importancia en la evaluación de genotipos desarrollados para diferentes condiciones de producción, es necesario integrar los conceptos de estabilidad (Lin et al. 1986; Lin y Binns 1994), para definir el comportamiento de los cultivares evaluados a través de ambientes contrastantes. La caracterización de la estabilidad de los genotipos evaluados en los diferentes sitios y años se efectuó usando la magnitud de la diferencia de rendimiento de los híbridos respecto al testigo, a través de los modelos de regresión propuestos por Finlay y Wilkinson (1963), Eberhart y Russell (1966); además del modelo multiplicativo AMMI (Zobel et al. 1988). La ventaja práctica del análisis de confiabilidad basado en las diferencias de rendimiento genotipo respecto al testigo es que con estos datos se permite comparar los híbridos en una amplia gama de ambientes y además determinar la estabilidad relativa de los genotipos evaluados sin considerar el conjunto original de materiales porque utiliza la diferencia con respecto al testigo común.

\section{RESULTADOS Y DISCUSIÓN}

\section{Análisis de confiabilidad respecto al testigo}

Una de las metas del mejoramiento genético es la identificación de genotipos con características fisiológicas y morfológicas asociadas con altos rendimientos en condiciones favorables y desfavorables (Cecarellí 1989). Una vez identificados los genotipos superiores se procede a su recomendación. No obstante, la probabilidad de certeza estadística de los nuevos cultivares de superar en potencial a un testigo en determinados ambientes influye directamente en la decisión de los fitomejoradores y productores para seleccionar los nuevos cultivares.

Desde el inicio del proyecto de formación de híbridos nacionales en 1987, el cultivar X-304C, ha sido el testigo utilizado para comparar el progreso genético de los nuevos genotipos de maíz, este es un híbrido simple de la Pioneer con amplia adaptación y buen comportamiento en las condiciones prevalecientes en las zonas maiceras de Panamá, razón por la cual es el más popular entre los productores, a pesar de su antigüedad.

Los resultados del análisis de confiabilidad $\left(\mathrm{RN}_{\mathrm{i}}\right) \mathrm{o}$ probabilidad de respuesta normalizada para los ocho híbridos permitieron clasificarlos en tres categorías con respecto al testigo, los genotipos P-9490, HS-8 y X$1358 \mathrm{~K}$ presentaron buena confiabilidad $\left(0,8 \geq \mathrm{RN}_{\mathrm{i}}<0,9\right)$ las probabilidades normalizadas de estos tres híbridos significa que por lo menos en 8 de cada 10 localidades hay una respuesta superior de estos genotipos con respecto al testigo (Cuadro 2).

Entre tanto, los híbridos 3018, 3031 y P-9422 presentaron confiabilidad promedio $\left(0,6 \geq \mathrm{RN}_{\mathrm{i}}<0,8\right)$, y el P-8916 presentó baja confiabilidad $\left(0,5 \geq \mathrm{RN}_{\mathrm{i}}<0,6\right)$. El híbrido triple P-8916 presenta una confiabilidad por debajo de 0,6 representando un riesgo relativamente similar para el productor con respecto al X-304C, es decir, que el productor puede sembrar cualquiera de los dos genotipos con resultados muy similares.

El híbrido X-304C, ha demostrado ser un genotipo excepcional desde su introducción al país hace aproximadamente quince años. El análisis de confiabilidad 
Cuadro 2. Rendimiento promedio, diferencias en rendimiento, desviación estándar y confiabilidad en ocho híbridos de maíz. Panamá, 1989-2000,

\begin{tabular}{lcccc}
\hline Híbridos & $\begin{array}{c}\text { Rend. } \\
(\mathbf{t} / \mathbf{h a})\end{array}$ & $\begin{array}{c}\text { Diferencia } \\
(\mathbf{t} / \mathbf{h a})\end{array}$ & $\begin{array}{c}\text { Desv. est. } \\
(\mathbf{t} / \mathbf{h a})\end{array}$ & $\mathbf{R N}_{\mathbf{i}}>\mathbf{0}$ \\
\hline P-8916 & 4,988 & 0,280 & 1,284 & $0,586 \mathrm{a}$ \\
3018 & 5,033 & 0,325 & 1,302 & $0,600 \mathrm{~b}$ \\
3031 & 5,382 & 0,674 & 1,233 & $0,708 \mathrm{~b}$ \\
P-9422 & 5,612 & 0,904 & 1,342 & $0,750 \mathrm{~b}$ \\
X-1358K & 5,813 & 1,105 & 1,306 & $0,801 \mathrm{c}$ \\
HS-8 & 5,894 & 1,186 & 1,293 & $0,820 \mathrm{c}$ \\
P-9490 & 5,861 & 1,153 & 1,255 & $0,821 \mathrm{c}$ \\
X-304C & 4,708 & 0,000 & & \\
\hline
\end{tabular}

Tipo de Confiabilidad: a baja, b promedio, c buena

sugiere que los híbridos más antiguos como el P-8916 representaban mayor riesgo para el productor, mientras que los genotipos liberados a mediados de los 90's $(3018,3031)$ representan una mejoría en el rendimiento. Sin embargo, la última generación de genotipos liberados hace dos años o menos presentan una buena confiabilidad, denotando el progreso genético alcanzado con los nuevos híbridos tanto por la empresa privada como por el sector público, representado por el IDIAP. En el estudio de Córdova et al. (1993), las ganancias en rendimiento sobre el híbrido $\mathrm{H}-5$ fueron más impactantes, a las obtenidas en este estudio, al presentar genotipos como el H30 y HB85 con confiabilidad superior o sea $0,9 \geq \mathrm{RNi} \leq 1,0)$. Entre mayor es la diferencia de rendimiento entre el genotipo evaluado y el testigo, más elevada es la confiabilidad.

Córdova et al. (1993), también plantean la confiabilidad económica como un factor asociado al cambio tecnológico propuesto. No obstante, en el caso de la zona donde se realizó el estudio, el cambio del testigo X304C por los híbridos P-9490 y HS-8, significa una reducción del costo, ya que la semilla, de los híbridos triples es más barata, en comparación con los híbridos simples. El testigo y el X-1358K que son híbridos simples, tienen valor similar de la semilla.

\section{Estabilidad relativa respecto al testigo}

De acuerdo con Lin et al. (1986), los análisis de estabilidad representan una medida relativa que permite hacer inferencia solamente en función del grupo fijo de genotipos que fueron evaluados. Sin embargo, Eskridge et al. (1993), indican que la confiabilidad permite hacer inferencias amplias en relación con la estabilidad, ya que solamente depende del testigo común y de un genotipo específico y no depende de otros cultivares Según Eskridge y Mumm (1992), Eskridge et al. (1993), la confiabilidad es una medida relacionada con otros parámetros de estabilidad como el coeficiente de regresión b b de Finlay y Wilkinson (1963), la desviación del cuadrado medio $\mathrm{S}^{2}{ }_{\mathrm{i}}$ de Eberhart y Russell (1966) y la varianza de estabilidad de Shukla (1972). Córdova et al. (1993), indican que la confiabilidad es una medida que evalúa tanto las ganancias en rendimiento con respecto al testigo, así como la variabilidad de la respuesta en los diferentes ambientes.

Las curvas de dispersión y regresión lineal de las diferencias de rendimiento con respecto al testigo en función de la probabilidad normalizada de los tres cultivares con mayor confiabilidad se presenta en la Figura 1. En ésta se aprecia como los tres cultivares presentan patrones muy similares de respuesta en comparación con el testigo. También se encontró que los coeficientes de las ecuaciones de las regresiones son muy similares. Esta similitud sugiere que el híbrido X$304 \mathrm{C}$ es un testigo que ha permitido discriminar eficientemente los cultivares para la recomendación a nivel de productores.

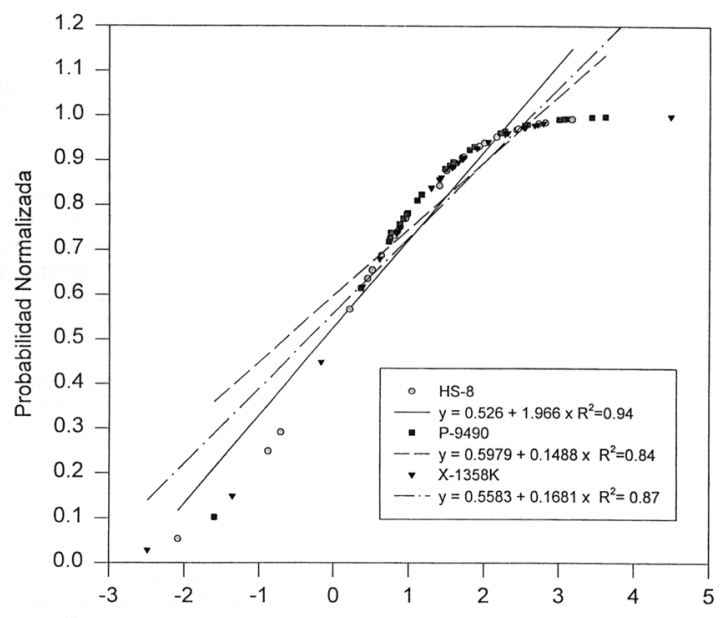

Diferencia de rendimiento con respecto al testigo ( $\mathrm{t} / \mathrm{ha})$

Figura 1. Relación de la confiabilidad con las diferencias de rendimiento de tres híbridos de maíz evaluados en Panamá.

Los resultados del análisis de estabilidad se presentan en el Cuadro 3. Podemos observar que los tres genotipos X-1358K, HS-8 y P-9490 mostraron buena estabilidad relativa respecto al X-304C. Siendo las estimaciones de los parámetros de estabilidad muy similares entre los tres modelos validados, denotando que además del avance genético en rendimiento de grano; 
Cuadro 3. Determinación de la estabilidad relativa los de tres híbridos con buena confiabilidad

\begin{tabular}{lccccc}
\hline Híbridos & $\mathbf{R N}_{\mathbf{i}}>\mathbf{0}$ & $\mathbf{b}_{\mathbf{i}}$ & $\mathbf{S}_{\mathbf{i}}$ & $\mathbf{R}^{\mathbf{2}}$ & AMMI \\
\hline X-1358K & 0,801 & 0,329 & 0,001 & 0,87 & $-0,879$ \\
HS-8 & 0,820 & $-0,635$ & 0,196 & 0,94 & $-0,240$ \\
P-9490 & 0,821 & 0,306 & 0,194 & 0,84 & $-0,861$ \\
\hline
\end{tabular}

también se observa un avance en el desarrollo de híbridos con mejor adaptabilidad y estabilidad en consecuencia con menos interacción con el ambiente.

Estos resultados coinciden con los obtenidos por Eskridge et al. (1993) y Córdova et al. (1993). Estos últimos determinaron que los cultivares HB-85 y H30 ambos con confiabilidad superior mostraron estabilidad relativa con respecto al $\mathrm{H} 5$. La consistencia de los resultados de este estudio, con los citados por la literatura confirman la relación entre la confiabilidad y los parámetros basados en la regresión lineal, que definen la estabilidad de los genotipos. El modelo AMMI, validado en este estudio, presenta puntuaciones que indican que los tres híbridos parecen adaptarse a ambientes desfavorables.

Por otro lado, los resultados de este estudio también sugieren que los híbridos HS-8, X-1358K y P9490 pueden ser usados como testigos en futuros ensayos de rendimiento en sustitución del actual testigo $\mathrm{X}-304 \mathrm{C}$.

\section{CONCLUSIONES}

El análisis de confiabilidad es una herramienta que mejora la certeza del investigador para recomendar nuevos cultivares.

El análisis de confiabilidad identificó progresos notables de los nuevos híbridos en relación a los rendimientos obtenidos.

Existe relación entre los parámetros de estabilidad utilizados con los resultados del análisis de confiabilidad.

\section{RECONOCIMIENTO}

Los autores dedican este trabajo como un homenaje póstumo a nuestro maestro y amigo inolvidable
Ingeniero Agronómo Alfonso Alvarado Dumont (1936-2000).

\section{LITERATURA CITADA}

BAKER, R.J. 1996 Recent research on genotype-environmental interaction. Paper to be presented at International Oat and Barley Conference. 8p.

CECCARELLI, S. 1989 Wide adaptation: How wide?. Euphytica 40:197-205

CÓRDOVA, H.S.; BARRETO, H.J.; CROSSA, J. 1993 Impacto del desarrollo de híbridos de maíz en Centro América: confiabilidad de las ganancias en rendimiento sobre el genotipo H5 y consideraciones para selección de testigos regionales. In: síntesis de resultados experimentales del PRM 1992. 4 : 3-10.

EBERHART, S.A.; RUSSELL, W.A. 1966. Stability parameters for comparing varieties. Crop Sci 6:36-40.

ESKRIDGE, K.M.; MUMM, R.F. 1992 Choosing plant cultivars based on the probability of outperforming a check. Theor. Appl. Genet. 84:494-500.

ESKRIDGE, K.M.; SMITH, O.S., BYRNE, P.F. 1993. Comparing test cultivars using reliability functions of testcheck differences from on-farm trials. Theor. Appl. Genet. 87:60-64

FINLAY, K.W.; WILKINSON, G.N. 1963 The analysis of adaptation in a plant-breeding programme. Aust. J. Agric. Res. 14:742-754.

JONES, T.A. 1988 A probability method for comparing varieties against checks. Crop Sci. 28:907-912.

LIN, C.S.; BINNS, M.R.; LEFKOVITCH, L.P. 1986. Stability analysis: Where do we stand? Crop Sci. 26:894-900.

LIN, C.S.; BINNS, M.R. 1994 Concepts and methods for analyzing regional trial data for cultivar and location selection. Plant Breeding Reviews 12:271-297.

SHUKLA GK. 1972. Some statistical aspects of partitioning genotype environmental components of variability. Heredity 29:237-245.

ZOBEL, R.W.; MADISON, J.W.; GAUCH, H.G. Jr. 1988 Statistical Analysis of a Yield Trial. Agron. J. 80:388393. 